\title{
Sharp Beckner-type inequalities for Cauchy and spherical distributions
}

\author{
Dominique Bakry* Ivan Gentil ${ }^{\dagger}$ and Grégory Scheffer ${ }^{\ddagger}$
}

December 18, 2018

\begin{abstract}
Using some harmonic extensions on the upper-half plane, and probabilistic representations, and curvature-dimension inequalities with some negative dimensions, we obtain some new optimal functional inequalities of the Beckner type for the Cauchy type distributions on the Euclidean space. These optimal inequalities appear to be equivalent to some non tight optimal Beckner inequalities on the sphere, and the family appears to be a new form of the Sobolev inequality.
\end{abstract}

Key words: Cauchy distribution, Beckner inequality, curvature-dimension condition, Poincaré inequality, Spherical analysis, Bessel processes, Stochastic calculus.

Mathematics Subject Classification (2010): 60G10, 60-XX, 58-XX.

\section{Introduction}

The so-called $\Gamma_{2}$ criterium is a way to prove functional inequalities such as Poincaré or logarithmic Sobolev inequalities. For instance, let $\Psi: \mathbb{R}^{d} \mapsto \mathbb{R}$ be a smooth function such that $\nabla^{2} \psi \geqslant \rho$ I with $\rho>0$, then the probability measure

$$
d \mu_{\psi}=\frac{e^{-\psi}}{Z} d x
$$

where $Z$ is the normalization constant which turns $\mu_{\psi}$ into a probability, satisfies both the Poincaré inequality,

$$
\int f^{2} d \mu_{\psi}-\left(\int f d \mu_{\psi}\right)^{2} \leq \frac{1}{\rho} \int|\nabla f|^{2} d \mu_{\psi}
$$

and the logarithmic Sobolev inequality

$$
\int f^{2} \log f^{2} d \mu_{\psi}-\int f^{2} d \mu_{\psi} \log \int f^{2} d \mu_{\psi} \leq \frac{2}{\rho} \int|\nabla f|^{2} d \mu_{\psi},
$$

*Institut de Mathématiques de Toulouse, Umr Cnrs 5219, Université de Toulouse - Paul Sabatier, bakry@math.univtoulouse.fr

${ }^{\dagger}$ Univ Lyon, Université Claude Bernard Lyon 1, CNRS UMR 5208, Institut Camille Jordan, 43 blvd. du 11 novembre 1918, F-69622 Villeurbanne cedex, France. gentil@math.univ-lyon1.fr

‡SCOR Investment Partners 5, avenue Kléber, 75795 Paris cedex 16, gregory.scheffer@gmail.com 
for any smooth function $f$. More generally, the same hypothesis leads to a family of interpolation inequalities between Poincaré and logarithmic Sobolev, namely, for any $p \in(1,2]$,

$$
\frac{p}{p-1}\left(\int f^{2} d \mu_{\psi}-\left(\int|f|^{2 / p} d \mu_{\psi}\right)^{p}\right) \leq \frac{2}{\rho} \int|\nabla f|^{2} d \mu_{\psi}
$$

where the case $p=2$ corresponds to the Poincaré inequality and the limit $p \rightarrow 1$ to the logarithmic Sobolev inequality. One of the technics used to prove such inequalities are based on the $\Gamma_{2}$ calculus (the $C D(\rho,+\infty)$ condition) introduced by the first author with M. Émery in [BÉ85] (see also [BGL14]). This family is the so-called Beckner inequalities, proved for the usual Gaussian measure, that is when $\psi=\frac{\|x\|^{2}}{2}$, by W. Beckner in [Bec89] (in that case $\rho=1$ ). Some improvements of such inequalities can be found in [AD05, DNS08, BG10]. Let use note also that recent developments for the Beckner inequalities applied to the Gaussian distribution in an optimal way have been proved in [IV17], also for uniformly strictly log-concave measures. Let us finish to quote the recent article of Nguyen [Ngu18] where the author generalizes some results of this paper with a completely different method, the method is extended later in [DGS18].

On the other hand, on the sphere $\mathbb{S}^{d} \subset \mathbb{R}^{d}$, with similar arguments, the following Beckner inequalities can be stated, $p \in(1,2]$,

$$
\frac{p}{p-1}\left(\int f^{2} d \mu_{\mathbb{S}}-\left(\int|f|^{2 / p} d \mu_{\mathbb{S}}\right)^{p}\right) \leq \frac{2}{d} \int|\nabla f|^{2} d \mu_{\mathbb{S}}
$$

see [BGL14], where in this situation, $\rho=d-1$.

It is important to notice that all Beckner inequalities (for the spherical and the Gaussian models) are optimal in the sense that constants in front of the right hand side are optimal. However, only the constant functions saturate these inequalities when $p \neq 2$.

The aim of this article is two-fold. First, we explain and improve in a general context the method introduced by the last author [Sch03] to obtain some new optimal inequalities. This method is mainly probabilistic, and makes strong use of various processes associated with the underlying structure behind the inequalities. He used this method to obtain an optimal Poincaré inequality under a $C D(\rho, n)$ condition, which is a stronger form of the condition $\nabla^{2} \psi \geqslant \rho I d$, and without integration by parts. More precisely, the method is enough robust to be applied to a nonsymmetric operator even if our example is the usual Laplacian which is symmetric. This method is rather technical and is based on the $C D(\rho, n)$ condition with a negative dimension $n$, together with a construction of some sub-harmonic functional. Let us note that negative dimension on the curvature-dimension $C D(\rho, n)$ condition has been introduced and used in [Sch03, Oht16, Mil17]. Stochastic calculus is also a main tool of our approach.

Secondly, improving unpublished results of the last author, we apply this method to obtain Beckner inequalities for the generalized Cauchy distribution, that is the probability measure on $\mathbb{R}^{d}$ with density

$$
d \nu_{b}(x)=\frac{1}{Z\left(1+|x|^{2}\right)^{b}} d x
$$

where $b>d / 2$ and $Z$ is the normalization constant. This measure does not satisfy any $C D(\rho,+\infty)$ condition with $\rho \geqslant 0$ but some $C D(0, n)$ condition with $n<0$, as will be explained with in detail in the paper. We prove the following new optimal family of inequalities,

$$
\frac{p}{p-1}\left[\int f^{2} d \nu_{b}-\left(\int|f|^{2 / p} d \nu_{b}\right)^{p}\right] \leq \frac{1}{(b-1)} \int \Gamma(f)\left(1+|y|^{2}\right) d \nu_{b}
$$


where $b \geqslant d+1$ and $p \in[1+1 /(b-d), 2]$.

Of course, when $p=2$ we recover a weighted Poincaré inequality for the Cauchy distribution proved in many papers, see [Sch01, $\mathrm{BBD}^{+} 07, \mathrm{BDGV10}$, Ngu14].

But more surprisingly, this family is equivalent to the following one on the sphere $\mathbb{S}^{d}$,

$$
\int h^{2} d \mu_{\mathbb{S}} \leq A\left(\int|h|^{2 / p} d \mu_{\mathbb{S}}\right)^{p}+\frac{16}{(m+2-d)(3 d-2+m)} \int \Gamma_{\mathbb{S}}(h) d \mu_{\mathbb{S}},
$$

where

$$
m \geqslant d+2, \quad p=1+\frac{2}{m-d} \in[1,2]
$$

and $A \geqslant 1$ is some explicit constant to be described below, converging to 1 as $p$ converges to 1 , or equivalently when $m$ converges to infinity. Inequality (1) is a tight inequality, that is constant functions are optimal, on the other hand inequality (2) is a non-tight inequality. On the other hand, this inequality (2) is optimal with explicit extremal functions. Moreover this new family of inequalities, or more precisely its behavior when $p$ converges to 1 , appears as a new form of a Sobolev inequality on the sphere, as many other ones described for example in [BCLS95].

The paper is organized as follows. In the next section, we describe the curvature-dimension condition $C D(\rho, n)$, even for negative $n$, and derive a general form of associated sub-harmonic functionals. In Section 3, we define an operator related to the generalized Cauchy distribution, and show that it satisfies the generalized curvature-dimension condition. In Section 4, we prove the new Beckner inequalities for these generalized Cauchy distribution. Finally, in Section 5, we prove the non-tight Becker inequalities on the $d$-dimensional sphere and show how they relate to the Sobolev inequality.

Notations: In all this paper, $d$ will be the dimension of the main space, and satisfies $d \geqslant 1$, and $d \geqslant 2$ in sections 4.2 and 5 . For every $X, Y \in \mathbb{R}^{d}, X \cdot Y=\sum_{i=1}^{d} X_{i} Y_{i}$ is the usual scalar product and $|X|^{2}$ is the Euclidean norm in $\mathbb{R}^{d}$.

\section{General properties on curvature-dimension condi- tion}

Let $\left(M^{d}, \mathfrak{g}\right)$ be a smooth connected $d$-dimensional Riemannian manifold and $\Delta_{\mathfrak{g}}$ its LaplaceBeltrami operator associated. The associated Ricci tensor is denoted Ric $\mathrm{R}_{\mathfrak{g}}$. For any diffusion operator $L$ on $M$ (that is a second order semi-elliptic differential operator with no zero-order term, see [BGL14]) we define the so-called carré du champ operator

$$
\Gamma^{L}(f, g)=\frac{1}{2}[L(f g)-f L g-g L f]
$$

with $\Gamma^{L}(f, f)=\Gamma^{L}(f)$ and its iterated operator

$$
\Gamma_{2}^{L}(f, f)=\Gamma_{2}^{L}(f)=\frac{1}{2} L\left(\Gamma_{L}(f)\right)-\Gamma^{L}(f, L f),
$$

for any smooth functions $f$ and $g$. We note $\Gamma\left(\right.$ resp. $\left.\Gamma_{2}\right)$ instead $\Gamma^{L}\left(\right.$ resp. $\left.\Gamma_{2}^{L}\right)$ when there is no possible confusion. 


\subsection{Definitions}

Definition $1\left(C D(\rho, n)\right.$ condition) An operator $L=\Delta_{\mathfrak{g}}+X$ with $X$ a smooth vector field satisfies a $C D(\rho, n)$ condition with $\rho \in \mathbb{R}$ and $n \in \mathbb{R} \backslash[0, d)$ if

$$
\Gamma_{2}(f) \geqslant \rho \Gamma(f)+\frac{1}{n}(L f)^{2},
$$

for any smooth function $f$.

As we shall see of the proof in Lemma 2, this condition will never hold when $n \in[0, d)$ but we will extend it for $n=0$.

Lemma 2 ([Bak94]) For any $\rho \in \mathbb{R}$ and $n \notin[0, d]$ the operator $L=\Delta_{\mathfrak{g}}+X$ satisfies a $C D(\rho, n)$ if and only if

$$
\frac{n-d}{n}(\operatorname{Ric}(L)-\rho \mathfrak{g}) \geqslant \frac{X \otimes X}{n},
$$

and when $n=d$ it reduces only to $X=0$ and the condition becomes $\operatorname{Ric}_{\mathfrak{g}} \geqslant \rho \mathfrak{g}$. In the definition, $\operatorname{Ric}(L)=\operatorname{Ric}_{\mathfrak{g}}-\nabla_{S} X$ where $\nabla_{S} X$ is the symmetrized tensor of $\nabla X$.

Proof. - For completeness, we give a sketch of proof of this result which can be helpful for the rest of the paper. For any smooth function $f$, the Bochner-Lichnerowicz-Weitzenbock formula states that (cf. [BGL14, Sec C.5]),

$$
\Gamma_{2}(f)=\left\|\nabla^{2} f\right\|_{H . S .}^{2}+\operatorname{Ric}_{\mathfrak{g}}(\nabla f, \nabla f)-\nabla X_{S}(\nabla f, \nabla f)=\left\|\nabla^{2} f\right\|_{H . S .}^{2}+\operatorname{Ric}(L)(\nabla f, \nabla f),
$$

where $\left\|\nabla^{2} f\right\|_{H . S}$. is the Hilbert-Schmidt norm of $f$. On a fixed point $x \in M$, we choose a local chart such that the metric $\mathfrak{g}=\left(\mathfrak{g}_{i, j}\right)$ is the identity matrix at the point $x$. In that case, $\nabla^{2} f=\left(\nabla_{i, j} f\right)$ is a $d$-dimensional symmetric matrix denoted $Y, \Gamma(f)=\sum_{i=1}^{d}\left(\partial_{i} f\right)^{2}$ and let denote $Z=\nabla f$. Since $\sum_{i, j=1}^{d} Y_{i, j}^{2}=\operatorname{tr}\left(Y^{2}\right)$, the $C D(\rho, n)$ condition (3) becomes

$$
\operatorname{tr}\left(Y^{2}\right)+Z \cdot R Z \geqslant \rho Z \cdot Z+\frac{1}{n}(\operatorname{tr}(Y)+X \cdot Z)^{2},
$$

where $R$ is a matrix representing the coordinates of the tensor $\operatorname{Ric}(L)$ at $x$. One can choose a new base in $\mathbb{R}^{d}$ such that $Y$ is diagonal. If we denote again $Z$ in the new base in $\mathbb{R}^{d}$, we have to prove such inequality, for every vectors $\left(Y_{i i}\right)_{1 \leq i \leq d}$ and $\left(Z_{i}\right)_{1 \leq i \leq d}$,

$$
\sum_{i=1}^{d} Y_{i i}^{2}+Z \cdot R Z \geqslant \rho Z \cdot Z+\frac{1}{n}\left(\sum_{i=1}^{d} Y_{i i}+X \cdot Z\right)^{2} .
$$

By taking $Y_{i i}$ all equals, from $\sum_{i=1}^{d} Y_{i i}^{2} \geqslant \frac{1}{d}\left(\sum_{i=1}^{d} Y_{i i}\right)^{2}$, the previous inequality is equivalent to

$$
\frac{1}{d}\left(\sum_{i=1}^{d} Y_{i i}\right)^{2}+Z \cdot R Z \geqslant \rho Z \cdot Z+\frac{1}{n}\left(\sum_{i=1}^{d} Y_{i i}+X \cdot Z\right)^{2} .
$$

Let $y=\sum_{i=1}^{d} Y_{i i}$, then the inequality becomes

$$
\frac{y^{2}}{d}+Z \cdot R Z \geqslant \rho Z \cdot Z+\frac{1}{n}(y+X \cdot Z)^{2} .
$$


In other words, the $C D(\rho, n)$ condition is satisfied if and only if, for every $y \in \mathbb{R}$ and, $Z \in \mathbb{R}^{d}$

$$
y^{2}\left(\frac{1}{d}-\frac{1}{n}\right)-y \frac{2}{n} X \cdot Z+Z \cdot(R-\rho \mathrm{Id}) Z-\frac{1}{n}(X \cdot Z)^{2} \geqslant 0 .
$$

Then, either $n=d$, then we need to assume that $X=0$ and $Z \cdot(R-\rho \mathrm{Id}) Z \geqslant 0$, either $\frac{1}{d}-\frac{1}{n}>0$ (with translates to $n \notin[0, d]$ ) and for any vector $Z$,

$$
\frac{(X \cdot Z)^{2}}{n} \leq \frac{n-d}{n} Z \cdot(R-\rho \mathrm{Id}) Z
$$

that is condition (4).

One may know define the tensor curvature-dimension condition, $T C D(\rho, n)$.

Definition $3(T C D(\rho, n)$ condition and quasi-models $Q M(\rho, n))$ Let $\rho \in \mathbb{R}, n \in \mathbb{R} \backslash\{d\}$ and an operator $L=\Delta_{\mathfrak{g}}+X$ with $X$ a smooth vector field.

- L satisfies a $T C D(\rho, n)$ condition if

$$
\frac{n-d}{n}(\operatorname{Ric}(L)-\rho \mathfrak{g}) \geqslant \frac{X \otimes X}{n},
$$

when $n \neq 0$, and

$$
-d(\operatorname{Ric}(L)-\rho \mathfrak{g}) \leq X \otimes X,
$$

when $n=0$.

- The operator $L$ is a quasi model $Q M(\rho, n)$ if

$$
(n-d)(\operatorname{Ric}(L)-\rho \mathfrak{g})=X \otimes X .
$$

Indeed, this new definition allows us to extend (3) when $n \in[0, d)$, and we shall see later crucial examples of such operators, which are even quasi-models.

\subsection{Sub-harmonic functionals}

In this section, we construct sub-harmonic functionals from harmonic functions under $T C D(\rho, n)$. We systematically explore the most general ones, in view of further use.

The next result is rather technical, and we shall see in the next sections how it leads to Poincaré inequalities or Beckner inequalities.

Theorem 4 (Sub-harmonic functionals under $T C D(\rho, n)$ or $Q M(\rho, n)$ )

Let $\Phi: I \times[0, \infty) \mapsto \mathbb{R}$ be a smooth function (I open interval of $\mathbb{R}$ ), and denote $\Phi_{i}$ and $\Phi_{i j}$ its first and second derivatives, $i, j \in\{1,2\}$. Let $L=\Delta_{\mathfrak{g}}+X$, where $X$ is a smooth vector field.

Let $\rho \in \mathbb{R}$ and $n \in \mathbb{R} \backslash\{d\}$. We assume that L satisfies the $T C D(\rho, n)$ condition with $n \in \mathbb{R} \backslash(0, d]$ (or the $Q M(\rho, n)$ condition). Then, if $F: M \mapsto I$ is smooth and satisfies $L(F)=0$, then $L[\Phi(F, \Gamma(F))] \geqslant 0$ as soon as $\Phi$ satisfies at any point $(y, z) \in I \times[0, \infty)$, either

$$
\left\{\begin{array}{l}
\Phi_{2}(y, z)>0 \\
\Phi_{2}(y, z) \frac{n+1-d}{n-d}+2 z \Phi_{22}(y, z)>0 \\
(n-d)\left(n \Phi_{2}(y, z)+2(n-1) z \Phi_{22}(y, z)\right)>0 ; \\
2 \rho \Phi_{2}(y, z)+\Phi_{11}(y, z)-2(n-1) \frac{z \Phi_{12}^{2}(y, z)}{n \Phi_{2}(y, z)+2(n-1) z \Phi_{22}(y, z)} \geqslant 0,
\end{array}\right.
$$


either

$$
\left\{\begin{array}{l}
\Phi_{2}(y, z)=0 \\
z \Phi_{22}(y, z)=0 \\
z \Phi_{12}(y, z)=0 \\
z \Phi_{11}(y, z) \geqslant 0
\end{array}\right.
$$

either

$$
\left\{\begin{array}{l}
\Phi_{2}(y, z)=0 \\
z \Phi_{22}(y, z)>0 \\
\Phi_{11}(y, z) \Phi_{22}(y, z)-\Phi_{12}^{2}(y, z) \geqslant 0
\end{array}\right.
$$

Remarks 5 - For simplicity, we omit the variables $y$ and $z$ inside the function $\Phi$. When $n \notin[0, d]$, condition $\Phi_{2} \frac{n+1-d}{n-d}+2 \Phi_{22} z>0$ is contained into $\Phi_{2}+2 \frac{n-1}{n} z \Phi_{22}>0$. Hence, provided that $n \notin[0, d]$, then (8) boils down to

$$
\left\{\begin{array}{l}
\Phi_{2}>0 \\
\Phi_{2}+2 \frac{n-1}{n} z \Phi_{22}>0 \\
2 \rho \Phi_{2}+\Phi_{11}-2(n-1) \frac{\Phi_{12}^{2} z}{n \Phi_{2}+2(n-1) \Phi_{22} z} \geqslant 0 .
\end{array}\right.
$$

It is interesting to notice that when $n \notin[0, d]$ the dimension $d$ does not appear in the previous conditions (11).

- As pointing out by a referee, condition (8) has a nice formulation by using a new variable coming from [IV18]. The authors set $M(y, u)=\Phi(x, \sqrt{u})$, then condition (8) is equivalent to, for any $(u, y) \in \mathbb{R} \times(0, \infty)$,

$$
(n-d)\left(\begin{array}{cc}
M_{11}(y, u)+\frac{\rho}{u} M_{12}(y, u) & M_{12}(y, u) \\
M_{12}(y, u) & M_{22}(y, u)+\frac{1}{(n-1) u} M_{2}(y, u)
\end{array}\right) \geqslant 0,
$$

together with the conditions $M_{2}(y, u)>0$ and $M_{22}(y, u)+\frac{1}{(n-1) u} M_{2}(y, u)>0$ where we use again the notation $M_{i j}=\partial_{i j}^{2} M$. Actually, we do not know implications of this new formulation of our condition (8).

Proof. - The proof is an improvement of the proof of Lemma 2.

If $L F=0$, then from the diffusion property of $L$ (cf. [BGL14, Sec 1.14]),

$$
\begin{array}{r}
L(\Phi(F, \Gamma(F)))=\Phi_{2} L \Gamma(F)+\Phi_{11} \Gamma(F)+2 \Phi_{12} \Gamma(F, \Gamma(F))+\Phi_{22} \Gamma(\Gamma(F), \Gamma(F)) \\
=2 \Phi_{2} \Gamma_{2}(F)+\Phi_{11} \Gamma(F)+2 \Phi_{12} \Gamma(F, \Gamma(F))+\Phi_{22} \Gamma(\Gamma(F), \Gamma(F)),
\end{array}
$$

since $L \Gamma(F)=2 \Gamma_{2}(F)$ when $L F=0$, for simplicity we note $\Phi_{i j}=\Phi_{i j}(F, \Gamma(F))$. At some point $x \in M$, we write all the expressions appearing in $L(\Phi(F, \Gamma(F)))$. From (5), they make use only of $\nabla \nabla F$ and $\nabla F$. We chose a system of coordinates in which at this point the metric $\mathfrak{g}$ is identity, $\nabla \nabla F$ is diagonal, with eigenvalues $\left(\lambda_{i}\right)_{1 \leq i \leq d}$. The components of $\nabla F$ are denoted $Z_{i}$, and the components of $X$ are denoted $-X_{i}$.

Since $L(F)=0$, the constraint can be written,

$$
\sum_{i=1}^{d} \lambda_{i}=\sum_{i=1}^{d} X_{i} Z_{i}
$$


and

$$
\Gamma(F, \Gamma(F))=2 \sum_{i=1}^{d} \lambda_{i} Z_{i}^{2}, \quad \Gamma(\Gamma(F), \Gamma(F))=4 \sum_{i=1}^{d} \lambda_{i}^{2} Z_{i}^{2}, \quad \Gamma(F)=\sum_{i=1}^{d} Z_{i}^{2} .
$$

From the $T C D(\rho, n)$ conditions and (5), the $\Gamma_{2}$ operator has a lower bound,

$$
\Gamma_{2}(F)=\sum_{i=1}^{d} \lambda_{i}^{2}+\operatorname{Ric}(L)(Z, Z) \geqslant \sum_{i=1}^{d} \lambda_{i}^{2}+\rho \sum_{i=1}^{d} Z_{i}^{2}+\frac{1}{n-d}\left(\sum_{i=1}^{d} X_{i} Z_{i}\right)^{2} .
$$

Under the $T C D(\rho, n)$ this is an inequality and we need to assume at this stage that $n \notin(0, d]$ and when the operator is a quasi-model $\operatorname{QM}(\rho, n)$ we only need to assume that $n \neq d$ and this is an equality.

Since $\Phi_{2} \geqslant 0$, from (12), we just have to minimize the following expression,

$$
\begin{aligned}
\frac{L(\Phi(F, \Gamma(F)))}{2} \geqslant 2 \Phi_{2}\left(\sum_{i=1}^{d} \lambda_{i}^{2}+\rho \sum_{i=1}^{d} Z_{i}^{2}+\right. & \left.\frac{1}{n-d}\left(\sum_{i=1}^{d} X_{i} Z_{i}\right)^{2}\right) \\
& +\Phi_{11} \sum_{i=1}^{d} Z_{i}^{2}+4 \Phi_{12} \sum_{i=1}^{d} \lambda_{i} Z_{i}^{2}+4 \Phi_{22} \sum_{i=1}^{d} \lambda_{i}^{2} Z_{i}^{2}
\end{aligned}
$$

for all $\left(\lambda_{i}\right)$ and $Z_{i}$, under the constraint $\sum_{i=1}^{d} \lambda_{i}=\sum_{i=1}^{d} X_{i} Z_{i}$.

Let $z=\sum_{i=1}^{d} Z_{i}^{2}=\Gamma(F)$ and $Z_{i}^{2}=z_{i}$, then the expression becomes by using the constraint (after dividing by 2 )

$$
\Phi_{2}\left(\sum_{i=1}^{d} \lambda_{i}^{2}+\rho z+\frac{1}{n-d}\left(\sum_{i=1}^{d} \lambda_{i}\right)^{2}\right)+\frac{\Phi_{11}}{2} z+2 \Phi_{12} \sum_{i=1}^{d} \lambda_{i} z_{i}+2 \Phi_{22} \sum_{i=1}^{d} \lambda_{i}^{2} z_{i} .
$$

We first optimise on the simplex $\left\{z_{i} \geqslant 0, \sum_{i=1}^{d} z_{i}=z\right\}$ where $z>0$ is fixed. Of course, we have to minimize along the simplex and the constraint $\left\{\sum_{i=1}^{d} \pm X_{i} \sqrt{z_{i}}=\sum_{i=1}^{d} \lambda_{i}\right\}$ which enlarge the set. We only minimize along the simplex which is lower. In fact it does not change the result and at this stage we can forget about the constraint. We first observe that the expression is affine in $z_{i}$. The vector $\left(z_{i}\right)_{1 \leq i \leq d}$ belongs to the simplex $\left\{z_{i} \geqslant 0, \sum_{i=1}^{d} z_{i}=z\right\}$, so that the extremals of this expression $\left(\left(\lambda_{i}\right)_{1 \leq i \leq d}\right.$ and $z>0$ are fixed $)$ is obtained at the one of the extreme point of the simplex. That is one of the $z_{i}$ is $z$ and all the other are 0 . Assuming that $z_{1}=z \neq 0$, one is now led to minimize

$$
\begin{aligned}
& \lambda_{1}^{2}\left(\Phi_{2}+2 \Phi_{22} z\right)+ 2 \Phi_{12} \lambda_{1} z+\Phi_{2} \sum_{i=2}^{d} \lambda_{i}^{2}+ \\
&=\lambda_{1}^{2}\left(\Phi_{2} \frac{n+1-d}{n-d}+2 \sum_{22}^{d} z\right)+\lambda_{1}\left(2 \Phi_{12} z+2 \frac{\Phi_{2}}{n-d} \sum_{i=2}^{d} \lambda_{i}\right) \\
&+\Phi_{2}\left(\sum_{i=2}^{d} \lambda_{i}^{2}+\frac{1}{n-d}\left(\sum_{i=2}^{d} \lambda_{i}\right)^{2}\right)+z\left(\Phi_{2} \rho+\frac{\Phi_{11}}{2}\right)
\end{aligned}
$$

Then, we minimize in $\lambda_{1}$. This imposes the second condition of (8), that is

$$
\Phi_{2} \frac{n+1-d}{n-d}+2 \Phi_{22} z>0,
$$


recall that $z=\Gamma(F)$. And the minimizer is given by

$$
\frac{L(\Phi(F, \Gamma(F)))}{2} \geqslant \Phi_{2}\left(\sum_{i=2}^{d} \lambda_{i}^{2}+\frac{1}{n-d}\left(\sum_{i=2}^{d} \lambda_{i}\right)^{2}\right)+z\left(\Phi_{2} \rho+\frac{\Phi_{11}}{2}\right)-\frac{\left(\Phi_{12} z+\frac{\Phi_{2}}{n-d} \sum_{i=2}^{d} \lambda_{i}\right)^{2}}{\Phi_{2} \frac{n+1-d}{n-d}+2 \Phi_{22} z}
$$

Let assume that $\Phi_{2}>0$. Decomposing the vector $\Lambda=\left(\lambda_{i}\right)_{2 \leq i \leq d}$ into a vector parallel to $(1, \cdots, 1)$ and a vector $u=\left(u_{i}\right)_{2 \leq i \leq d}$ orthogonal to it, that is $\Lambda=\lambda(1, \cdots, 1)+u$ with $\sum_{i=2}^{d} u_{i}=$ 0 . From this decomposition, we have $\sum_{i=2}^{d} \lambda_{i}=(d-1) \lambda$ and

$$
\sum_{i=2}^{d} \lambda_{i}^{2}=\sum_{i=2}^{d}\left(\lambda+u_{i}\right)^{2} \geqslant(d-1) \lambda^{2}
$$

We see that we need to find the minimum in $\lambda$ of

$$
\begin{gathered}
\Phi_{2}\left((d-1) \lambda^{2}+\frac{(d-1)^{2}}{n-d} \lambda^{2}\right)+z\left(\Phi_{2} \rho+\frac{\Phi_{11}}{2}\right)-\frac{\left(\Phi_{12} z+\frac{(d-1) \Phi_{2}}{n-d} \lambda\right)^{2}}{\Phi_{2} \frac{n+1-d}{n-d}+2 \Phi_{22} z} \\
=\lambda^{2} \frac{\Phi_{2}(d-1)\left(n \Phi_{2}+2(n-1) z \Phi_{22}\right)}{(n-d)\left(\Phi_{2} \frac{n+1-d}{n-d}+2 \Phi_{22} z\right)}-\lambda \frac{2(d-1) \Phi_{2} \Phi_{12} z}{(n-d)\left(\Phi_{2} \frac{n+1-d}{n-d}+2 \Phi_{22} z\right)} \\
-\frac{\Phi_{12}^{2} z^{2}}{\Phi_{2} \frac{n+1-d}{n-d}+2 \Phi_{22} z}+z\left(\Phi_{2} \rho+\frac{\Phi_{11}}{2}\right)
\end{gathered}
$$

Then, once again, to get a minimizer in $\lambda$, we also need

$$
(n-d)\left(n \Phi_{2}+2(n-1) \Phi_{22} z\right)>0,
$$

which is the third assumption in (8). Then, the minimizer is

$$
\begin{aligned}
& \frac{L(\Phi(F, \Gamma(F)))}{2} \geqslant-\frac{(d-1) \Phi_{2} \Phi_{12}^{2} z^{2}}{(n-d)\left(n \Phi_{2}+2(n-1) z \Phi_{22}\right)\left(\Phi_{2} \frac{n+1-d}{n-d}+2 \Phi_{22} z\right)} \\
& \quad-\frac{\Phi_{12}^{2} z^{2}}{\Phi_{2} \frac{n+1-d}{n-d}+2 \Phi_{22} z}+z\left(\Phi_{2} \rho+\frac{\Phi_{11}}{2}\right)=\frac{-z^{2}(n-1) \Phi_{12}^{2}}{n \Phi_{2}+2 z(n-1) \Phi_{22}}+z\left(\Phi_{2} \rho+\frac{\Phi_{11}}{2}\right) \geqslant 0,
\end{aligned}
$$

which is the fourth assumption in (8) since $z \geqslant 0$.

When $\Phi_{2}=0$ at some point $(y, z)$, we follow the inequality to check the assumptions needed.

Corollary 6 (Sub-harmonic functionals under $T C D(0, n)$ ) Let assume that $L=\Delta_{\mathfrak{g}}+X$ satisfies a $T C D(0, n)$ with $n<0$. Let $\theta$ be a positive and smooth function on an interval $I \subset \mathbb{R}$ such that

$$
2 \frac{n-1}{n}\left(\theta^{\prime}\right)^{2} \leq \theta \theta^{\prime \prime}
$$

Then for any function $F: M \mapsto I$ such that $L F=0$,

$$
L(\theta(F) \Gamma(F)) \geqslant 0 .
$$

In particular, for any $\beta \in[n /(2-n), 0]$ and nonnegative harmonic function $F$,

$$
L\left(F^{\beta} \Gamma(F)\right) \geqslant 0 .
$$


When $n$ goes to $-\infty$, the condition (13) on $\theta$ degenerates into $2 \theta^{\prime} \leq \theta \theta^{\prime \prime}$. This means that the function $\Phi$ such that $\Phi^{\prime \prime}=\theta$ is admissible : $\Phi$ is convex and $1 / \Phi^{\prime \prime}$ is concave.

Moreover, when $n \in(-\infty, 0)$, the extremal case is given when $\theta(x)=x^{n /(2-n)}$, where (13) is an equality.

Although we shall mainly use Corollary 6 instead the general result Theorem 4, the proof in this last case is not really simpler than the general one.

\section{The operators $Q_{t}^{(m)}$}

Definition 7 (The operators $Q_{t}^{(m)}$ ) For any $t \geqslant 0, m>0, x \in \mathbb{R}^{d}$, and any bounded function $f: \mathbb{R}^{d} \mapsto \mathbb{R}$, let

$$
Q_{t}^{(m)}(f)(x)=\frac{1}{c(m, d)} \int \frac{f(y t+x)}{\left(1+|y|^{2}\right)^{\frac{m+d}{2}}} d y,
$$

where

$$
c(m, d)=\int \frac{1}{\left(1+|y|^{2}\right)^{\frac{m+d}{2}}} d y=\frac{\Gamma\left(\frac{m}{2}\right)}{\Gamma\left(\frac{m+d}{2}\right)} \pi^{d / 2}
$$

is the normalization constant, such that $Q_{t}^{(m)}(1)=1$. In other words, for any $t>0$,

$$
Q_{t}^{(m)}(f)(x)=\int f(y) q_{t}(x, d y)
$$

where the kernel $q_{t}$ is given by

$$
q_{t}(x, d y)=\frac{1}{c(m, d)} \frac{t^{m}}{\left(t^{2}+|x-y|^{2}\right)^{\frac{m+d}{2}}} d y .
$$

The probability measure

$$
\frac{1}{c(m, d)}\left(1+|y|^{2}\right)^{-\frac{m+d}{2}} d y
$$

in $\mathbb{R}^{d}$, with $m>0$, is known in statistics as the multivariate $t$-distribution with $m$ degrees of freedom. There is a huge literature on the subject, see for instance [KN04].

Through an integration by parts (or from the computation of normalization constants) we may notice the useful formula,

$$
\frac{c(m, d)}{c(m-2, d)}=\frac{m-2}{m-2+d}
$$

for any $m>2$.

Proposition 8 (Harmonicity of $Q_{t}^{(m)} f$ ) Let $m>0$. For any smooth and compactly supported function $f$, the map $(0,+\infty) \times \mathbb{R}^{d} \ni(t, x) \mapsto Q_{t}^{(m)}(f)(x)$ is solution of the elliptic equation $\Delta^{(m)} Q_{t}^{(m)}(f)=0$ where

$$
\Delta^{(m)}=\Delta_{\mathbb{R}^{d}}+\partial_{t t}^{2}+\frac{1-m}{t} \partial_{t}
$$


Proof. - This can be proved directly on the expression of the kernel (17).

From Proposition 8 (or from a direct computation) the operators $\left(Q_{t}^{(m)}\right)_{t \geqslant 0}$ admit an another formulation. Let $\left(Z_{s}\right)_{s \geqslant 0}=\left(X_{s}, Y_{s}\right)_{s \geqslant 0} \in \mathbb{R}^{d} \times \mathbb{R}$ be a diffusion process with generator $\Delta^{(m)}$ starting from $(x, t) \in \mathbb{R}^{d} \times(0, \infty)$. That is $\left(X_{s}\right)_{s \geqslant 0}$ is a Brownian motion up to a factor $\sqrt{2}$ and $\left(Y_{s}\right)_{s \geqslant 0}$ is independent of $\left(X_{s}\right)_{s \geqslant 0}$ with generator $\partial_{t}^{2}+\frac{1-m}{t} \partial_{t}$.

The process $\left(Y_{s}\right)_{s \geqslant 0}$ is a Bessel process with parameter $m$ starting from $t>0$. When $m>0$, the stopping time $S=\inf \left\{s>0, Y_{s}=0\right\}$ is finite a.s., and since $Q_{t}^{(m)}(f)$ is a solution of the elliptic equation (15), from Itô's Lemma, or Dynkin's formula [Øk03, Sec 7.3]

$$
Q_{t}^{(m)}(f)(x)=E_{x, t}\left(f\left(X_{S}\right)\right)
$$

for every smooth and bounded function $f$. In other words since, $S$ and $\left(X_{s}\right)_{s \geqslant 0}$ are independent, if $\sigma_{m}(s, t) d s$ denotes the law of the hitting time $S$ starting from $t>0$,

$$
Q_{t}^{(m)}(f)(x)=\int_{0}^{\infty} P_{s} f(x) \sigma_{m}(s, t) d s,
$$

where $\left(P_{s}\right)_{s \geqslant 0}$ is the heat semi-group in $\mathbb{R}^{d}$ associated to the Euclidean Laplacian $\Delta$. From the definition of the operators $Q_{t}^{(m)}$ and the definition of the the equation $\left(P_{s}\right)_{s \geqslant 0}$,

$$
P_{s} f(x)=\int f(y) \exp \left(-\frac{|x-y|^{2}}{4 s}\right) \frac{d y}{(4 \pi s)^{d / 2}},
$$

and this gives a way to compute the law $\sigma_{m}$ of the hitting time. One obtains, for any $t>0$, the probability measure

$$
\sigma_{m}(s, t) d s=\frac{1}{2^{m} \Gamma(m / 2)} \frac{t^{m} \exp \left(-t^{2} / 4 s\right)}{s^{m / 2+1}} d s .
$$

This formula can also be found for instance in [BS96].

The map $(t, s) \mapsto \sigma_{m}(t, s)$ satisfies

$$
\partial_{s}=\partial_{t t}^{2}+\frac{1-m}{t} \partial_{t}
$$

with Dirichlet boundary conditions, $\sigma_{m}(t, 0)=0$ for every $t>0$, and $\sigma_{m}(0, s) d s=\delta_{0}(s)$. We recognize in the RHS the generator of the Bessel process. This phenomena is universal and has nothing to do in particular with Bessel processes, see for instance [Pav14, Sec 7.2].

From this observation and an integration by parts, one recovers that the map $(t, x) \mapsto$ $Q_{t}^{(m)} f(x)$ satisfies the elliptic equation $\Delta^{(m)} Q_{t}^{(m)}(f)=0$.

Lemma 9 Let $m>0$ and $p \in(0, m / 2)$, then for any smooth and bounded function $g$,

$$
E_{x, t}\left(S^{p} g\left(X_{S}\right)\right)=t^{2 p} \frac{\Gamma\left(\frac{m}{2}-p\right)}{4^{p} \Gamma\left(\frac{m}{2}\right)} Q_{t}^{(m-2 p)}(g)(x) .
$$

In particular, when $p=1$ and $m>2$,

$$
E_{x, t}\left(S g\left(X_{S}\right)\right)=\frac{t^{2}}{2(m-2)} Q_{t}^{(m-2)}(g)(x) .
$$


Proof. - The formula comes from a direct computation since we have

$$
\begin{aligned}
E_{x, t}\left(S^{p} g\left(X_{S}\right)\right)=\frac{1}{2^{m} \Gamma(m / 2)(4 \pi)^{d / 2}} \int g(y) \int_{0}^{\infty} & s^{p-\frac{m+d}{2}-1} \exp \left(-\frac{|x-y|^{2}}{4 s}-\frac{t^{2}}{4 s}\right) d s d y \\
& =t^{2 p} \frac{\Gamma\left(\frac{m}{2}-p\right)}{2^{m} \Gamma(m / 2)(4 \pi)^{d / 2}} \int P_{s} g(y) \sigma_{m-2 p}(s) d s .
\end{aligned}
$$

Lemma $10\left(Q M\right.$ condition for $\left.\Delta^{(m)}\right)$ For any $m \neq 1$, the operator $\Delta^{(m)}$ on $\mathbb{R}^{d} \times(0, \infty)$ is a quasi-model $Q M(0, d-m+2)$.

Proof. - For that model,

$$
\Delta^{(m)}=\Delta_{\mathbb{R}^{d+1}}+\frac{1-m}{t} \partial_{t}
$$

that is $X=\frac{1-m}{t} \partial_{t}$. Then $\operatorname{Ric}(L)=\frac{1-m}{t^{2}}\left(\partial_{t}\right)^{2}$ and $X \otimes X=\frac{(1-m)^{2}}{t^{2}}\left(\partial_{t}\right)^{2}$ and the result follows from the definition (7).

\section{Beckner and $\Phi$-entropy inequalities for Cauchy type distribution}

\subsection{Beckner inequalities for Cauchy type distribution}

Let $f: \mathbb{R} \mapsto[0, \infty)$ be a smooth and compactly supported function, and let $F(t, x)=Q_{t}^{(m)}(f)(x)$ with $m>0$.

Let $\beta \in \mathbb{R}$. From proposition (8), $F$ is $\Delta^{(m)}$-harmonic, and then

$$
\Delta^{(m)}\left(F^{\beta+2}\right)=(\beta+2)(\beta+1) F^{\beta} \Gamma^{\Delta^{(m)}}(F) .
$$

and, as in Section 3, we can write,

$$
E_{x, t}\left(F\left(Z_{S}\right)^{\beta+2}\right)=E_{x, t}\left(F\left(Z_{0}\right)^{\beta+2}\right)+(\beta+2)(\beta+1) \int_{0}^{\infty} E_{x, t}\left(F^{\beta}\left(Z_{s}\right) \Gamma^{\Delta^{(m)}}(F)\left(Z_{s}\right) 1_{s \leq S}\right) d s,
$$

where $\left(Z_{s}\right)_{s \geqslant 0}$ is the Markov process in $\mathbb{R}^{d+1}$ with generator $\Delta^{(m)}$. Since $F\left(Z_{S}\right)=f\left(X_{S}\right)$, from (20) we have the general equation,

$$
Q_{t}^{(m)}\left(f^{\beta+2}\right)=Q_{t}^{(m)}(f)^{\beta+2}+(\beta+2)(\beta+1) \int_{0}^{\infty} E_{x, t}\left(F^{\beta}\left(Z_{s}\right) \Gamma^{\Delta^{(m)}}(F)\left(Z_{s}\right) 1_{s \leq S}\right) d s .
$$

Again, from Dynkin's formula applied to $F^{\beta} \Gamma^{\Delta^{(m)}}(F)$, we have

$$
\begin{aligned}
& E_{x, t}\left(F^{\beta}\left(Z_{s}\right) \Gamma^{\Delta^{(m)}}(F)\left(Z_{s}\right) 1_{s \leq S}\right)= \\
& E_{x, t}\left(F^{\beta}\left(Z_{S}\right) \Gamma^{\Delta^{(m)}}(F)\left(Z_{S}\right) 1_{s \leq S}\right)-E_{x, t}\left(\int_{s}^{S} \Delta^{(m)}\left(F^{\beta} \Gamma^{\Delta^{(m)}}(F)\right)\left(Z_{u}\right) d u 1_{s \leq S}\right) .
\end{aligned}
$$


In other words,

$$
\begin{aligned}
Q_{t}^{(m)}\left(f^{\beta+2}\right)-Q_{t}^{(m)}(f)^{\beta+2} & =(\beta+2)(\beta+1) E_{x, t}\left(S F^{\beta}\left(Z_{S}\right) \Gamma^{\Delta^{(m)}}(F)\left(Z_{S}\right)\right) \\
& -(\beta+2)(\beta+1) \int_{0}^{\infty} E_{x, t}\left(\int_{s}^{S} \Delta^{(m)}\left(F^{\beta} \Gamma^{\Delta^{(m)}}(F)\right)\left(Z_{u}\right) d u 1_{s \leq S}\right) d s .
\end{aligned}
$$

Let now assume that $m \geqslant d+2$, and let denote $n=d-m+2(n \leq 0)$. Let also assume that $\beta \in\left[\frac{n}{2-n}, 0\right]$ (that is $\beta \in\left[-1+\frac{2}{m-d}, 0\right], \frac{2}{m-d}>0$ ). Since $n \leq 0, \Delta^{(m)}$ satisfies $\operatorname{TCD}(0, n)$ (Lemma 10) and from Corollary 6,

$$
\Delta^{(m)}\left(F^{\beta} \Gamma^{\Delta^{(m)}}(F)\right) \geqslant 0 .
$$

The equation (25) becomes

$$
Q_{t}^{(m)}\left(f^{\beta+2}\right)-Q_{t}^{(m)}(f)^{\beta+2} \leq(\beta+2)(\beta+1) \int_{0}^{\infty} E_{x, t}\left(F^{\beta}\left(Z_{S}\right) \Gamma^{\Delta^{(m)}}(F)\left(Z_{S}\right) \mathbb{1}_{s \leq S}\right) d s,
$$

which is

$$
Q_{t}^{(m)}\left(f^{\beta+2}\right)-Q_{t}^{(m)}(f)^{\beta+2} \leq(\beta+2)(\beta+1) E_{x, t}\left(S F^{\beta}\left(Z_{S}\right) \Gamma^{\Delta^{(m)}}(F)\left(Z_{S}\right)\right) .
$$

Starting form $Z_{0}=(x, t)$,

$$
F^{\beta}\left(Z_{S}\right) \Gamma^{\Delta^{(m)}}(F)\left(Z_{S}\right)=f^{\beta}\left(X_{S}\right) \Gamma^{\Delta^{(m)}}(F)\left(X_{S}, 0\right)=f^{\beta}\left(X_{S}\right)\left[\Gamma(f)\left(X_{S}\right)+\left(\partial_{t} F\right)^{2}\left(X_{S}, 0\right)\right],
$$

since $\Gamma^{\Delta^{(m)}}(F)=\Gamma(F)+\left(\partial_{t} F\right)^{2}$ where $\Gamma(f)=|\nabla f|^{2},\left(f: \mathbb{R}^{d} \mapsto \mathbb{R}\right)$ is the carré du champ operator associated to Euclidean Laplacian in $\mathbb{R}^{d}$. From Proposition 8 , since $m>1, \partial_{t} F(x, 0)=$ 0 then

$$
F^{\beta}\left(Z_{S}\right) \Gamma(F)\left(Z_{S}\right)=f^{\beta}\left(X_{S}\right) \Gamma(f)\left(X_{S}\right) .
$$

We can now apply Lemma 9, to get

$$
Q_{t}^{(m)}\left(f^{\beta+2}\right)-Q_{t}^{(m)}(f)^{\beta+2} \leq(\beta+2)(\beta+1) \frac{t^{2}}{2(m-2)} Q_{t}^{(m-2)}\left(f^{\beta} \Gamma(f)\right) .
$$

Changing $f^{\beta+2}$ by $f^{2}$ and $p=\beta+2$ we have obtained

Theorem 11 (Beckner inequalities for $Q_{t}^{(m)}$ ) For any $m \geqslant d+2$ and $p \in\left[1+\frac{2}{m-d}, 2\right]$, and for any smooth function $f \geqslant 0$,

$$
\frac{p}{p-1}\left(Q_{t}^{(m)}\left(f^{2}\right)-Q_{t}^{(m)}\left(f^{2 / p}\right)^{p}\right) \leq \frac{2 t^{2}}{m-2} Q_{t}^{(m-2)}(\Gamma(f))
$$

When $p=2(m \geqslant d+2)$, we obtain the Poincaré inequality for every smooth function $f$,

$$
Q_{t}^{(m)}\left(f^{2}\right)-Q_{t}^{(m)}(f)^{2} \leq \frac{t^{2}}{(m-2)} Q_{t}^{(m-2)}(\Gamma(f)) .
$$

For $t=1$ and $x=0$ in the previous inequality, we have obtained the Beckner inequality for the Cauchy distribution $\frac{1}{c(m, d)} \frac{1}{\left(1+\left|y^{2}\right|\right)^{\frac{m+d}{2}}}$. 
So, for any $m \geqslant d+2$ and $p \in\left[1+\frac{2}{m-d}, 2\right]$ one has,

$$
\begin{aligned}
\frac{p}{p-1}\left[\frac{1}{c(m, d)} \int \frac{f^{2}}{\left(1+|y|^{2}\right)^{\frac{m+d}{2}}} d y-\left(\frac{1}{c(m, d)} \int \frac{f^{2 / p}}{\left(1+|y|^{2}\right)^{\frac{m+d}{2}}} d y\right)^{p}\right] \\
\leq \frac{2}{m-2} \frac{1}{c(m-2, d)} \int \frac{\Gamma(f)}{\left(1+|y|^{2}\right)^{\frac{m+d-2}{2}}} d y .
\end{aligned}
$$

The inequality can be written as a weighted Poincaré-type inequality for a generalized Cauchy distribution. Let define for $b>\frac{d}{2}$, the probability measure

$$
d \nu_{b}(y)=\frac{1}{c(2 b-d, d)} \frac{1}{\left(1+|y|^{2}\right)^{b}} d y
$$

and replacing $b=(m+d) / 2$ in the previous inequality, we have obtained, using (18),

Theorem 12 (Beckner inequalities for Cauchy distribution) For any $b \geqslant d+1$ and $p \in$ $\left[1+\frac{1}{b-d}, 2\right]$,

$$
\frac{p}{p-1}\left[\int f^{2} d \nu_{b}-\left(\int f^{2 / p} d \nu_{b}\right)^{p}\right] \leq \frac{1}{(b-1)} \int \Gamma(f)\left(1+|y|^{2}\right) d \nu_{b}
$$

for any smooth and nonnegative function $f$.

Moreover, the inequality is optimal, that is, fixing $p \in\left[1+\frac{1}{b-d}, 2\right]$, the constant $\frac{1}{(b-1)}$ is the best possible in the inequality (30).

The case $p=2$ can be extended to any smooth function $f$ (not only nonnegative function). Then we obtain the following inequality, proved in [Sch01, $\left.\mathrm{BBD}^{+} 07, \mathrm{BDGV10}, \mathrm{Ngu14}\right]$.

Corollary 13 (Poincaré inequality for Cauchy distribution) For any $b \geqslant d+1$, the measure $\nu_{b}$ satisfies a Poincaré type inequality,

$$
\int f^{2} d \nu_{b}-\left(\int f d \nu_{b}\right)^{2} \leq \frac{1}{2(b-1)} \int \Gamma(f)\left(1+|y|^{2}\right) d \nu_{b}
$$

for any smooth function $f$. Moreover, functions $y \mapsto y_{i}$ with $1 \leq i \leq d$ are extremal functions.

Proof. - We only have to prove optimality. Apply the Poincaré inequality (31) to the function $y \mapsto y_{i}$ for some $1 \leq i \leq d$,

$$
\nu_{b}\left(y_{i}^{2}\right) \leq \frac{1}{2(b-1)} \nu_{b}\left(1+|y|^{2}\right)=\frac{1}{2(b-1)}\left(1+\nu_{b}\left(|y|^{2}\right)\right),
$$

or equivalently

$$
\frac{1}{d} \nu_{b}\left(|y|^{2}\right) \leq \frac{1}{2(b-1)}\left(1+\nu_{b}\left(|y|^{2}\right)\right)
$$

But this last one is an equality since, from (18), $\nu_{b}\left(|y|^{2}\right)=\frac{d}{2 b-2-d}$.

\section{Remarks 14}

- Inequality (30) is equivalent to (27); indeed we only have to replace the map $y \mapsto f(y)$ by $y \mapsto f(t y+x)$. 
- We cannot reach the logarithmic Sobolev inequality, since in our computation $p \geqslant 1+1 / b$ and this inequality would require a limit p goes to 1.

- A Taylor expansion with $\varepsilon \rightarrow 0$ in inequality (30) with $f=1+\varepsilon g$ implies back the optimal Poincaré inequality (31), since

$$
\frac{p}{p-1}\left[\int f^{2} d \nu_{b}-\left(\int f^{2 / p} d \nu_{b}\right)^{p}\right]=2 \varepsilon^{2}\left[\int g^{2} d \nu_{b}-\left(\int g d \nu_{b}\right)^{2}\right]+o\left(\varepsilon^{2}\right) .
$$

We do not know if there are extremal functions apart of constant functions.

- Corollary 13 has also been proved by Nguyen [Ngu14, Cor. 14], using the Brunn-Minkowski theory (see also the approach in [BL09, ABJ18]).

In the one dimensional case, the exact value has been computed by Bonnefont, Joulin and Ma in [BJM16, Thm 3.1]. Their result is more general, since they compute the optimal constant for every $b>1 / 2$. The constant of (31) with $d=1$ is

$$
\left\{\begin{array}{l}
\frac{1}{2(b-1)} \quad \text { if } \quad b \geqslant 3 / 2 \\
\frac{4}{(2 b-1)^{2}} \quad \text { if } \quad 1 / 2<b \leq 3 / 2 .
\end{array}\right.
$$

It is interesting to notice that there are two regimes, depending on the range of the parameter $b$. Actually, we are not able with our method to reach this range, even in dimension 1.

- Applying inequality (30) to the function $x \mapsto f(\sqrt{2 b} x)$, then, when $b \rightarrow+\infty$, the inequality becomes the optimal Beckner inequality for the Gaussian measure $\gamma$ in $\mathbb{R}^{d}$,

$$
\frac{p}{p-1}\left[\int f^{2} d \gamma-\left(\int f^{2 / p} d \gamma\right)^{p}\right] \leq 2 \int \Gamma(f) d \gamma
$$

for any smooth and positive function $f$. This inequality is proved by W. Beckner [Bec89]. In that case the inequality holds for any $p \in(1,2]$, and the limit case (when $p \rightarrow 1$ ) leads to the optimal Logarithmic Sobolev inequality for the Gaussian measure.

- At the same time, Nguyen proves in [Ngu18] the Beckner inequality (30) for a range of parameter $p$ strictly contained in our interval $[1+1 /(b-d), 2]$. Even if, with his method, the parameter $p$ can not reach the full interval, Nguyen is able to extend the result to a general class of probability measures with a convexity assumption. The method is actually improved in a more general context in [DGS18].

\subsection{Tightness of the inequalities}

We are interesting in the tightness of inequality (27), in the sense of the curvature-dimension condition. In all this section we assume that $d \geqslant 2$.

Lemma 15 (Taylor expansion of $\left(Q_{t}^{(m)}\right)$ For any $m>4$, we have

$$
Q_{t}^{(m)}=\mathrm{Id}+\frac{\Delta}{2(m-2)} t^{2}+\frac{\Delta^{2}}{8(m-2)(m-4)} t^{4}+o\left(t^{4}\right) .
$$

The formula has to be understood as follow, for any smooth and compactly supported function $f$, the rest function $o\left(t^{4}\right)$ is uniformly bounded in the variable $x$. 
Proof. - One can check directly on the formula. On the other hand, one can deduce the formula with functional analysis, at least formally. Since $Q_{t}^{(m)}$ is $\Delta^{(m)}$-harmonic from Proposition 8 then, from the functional analysis point of view, $Q_{t}^{(m)}$ is a function $G_{m}(t, \Delta)$. From the definition (19) of $\Delta^{(m)}$, the function $G_{m}$ satisfies for $t \geqslant 0, x<0$,

$$
x G_{m}(t, x)+\frac{1-m}{t} \partial_{t} G_{m}(t, x)+\partial_{t t}^{2} G_{m}(t, x)=0,
$$

with the boundary conditions $G_{m}(0, x)=1$. Solution of such differential equation is given by, $G_{m}(t, x)=H_{m}\left(-t^{2} x\right)$ where $H_{m}$ satisfies for any $\lambda \geqslant 0$,

$$
4 \lambda H_{m}^{\prime \prime}(\lambda)-2(m-2) H_{m}^{\prime}(\lambda)-H_{m}(\lambda)=0 .
$$

An asymptotic development of $H_{m}(\lambda)=\sum_{p \geqslant 0} c_{p} \lambda^{p}$ gives $c_{0}=1\left(\right.$ since $G_{m}(0, x)=1$ ) and

$$
c_{p+1}=\frac{1}{2(p+1)(2 p-m+2)} c_{p} .
$$

For the forth order Taylor expansion of $Q_{t}^{(m)}$, we get (32), that is $c_{1}=\frac{1}{2(2-m)}$ and $c_{2}=$ $\frac{1}{8(m-4)(m-2)}$.

This Lemma is fundamental. The Taylor expansion in (27), or equivalently in (28), gives in return the $C D(0, d)$ conditions. We say then that the inequalities are $\Gamma_{2}$-tight and nothing has been lost in term of curvature-dimension condition (3), in other words the Taylor expansion of the inequality implies the $C D(0, d)$ condition.

To see that, it is enough to compute the Taylor expansion of the inequality (27), when $t$ goes to 0 . It is important to fix $\beta=\frac{2-m+d}{m-d}=-1+\frac{2}{m-d}$ the extremal bound of the admissible interval of $\beta$.

It is clear that the zero order terms vanish, it is also not difficult to observe that the second order terms also vanish. Therefor we only have to compute the fourth order terms and we obtain the following expression,

$$
\begin{gathered}
\frac{1}{8(m-4)(m-2)} \Delta^{2}\left(f^{\beta+2}\right)-\frac{(\beta+2)}{8(m-4)(m-2)} f^{\beta-1} \Delta^{2}(f)-\frac{(\beta+2)(\beta+1)}{8(m-2)^{2}} f^{\beta-1} \Delta(f)^{2} \leq \\
\frac{\beta(\beta+1)(\beta+2)}{2(m-4)(m-2)} \Gamma(f, \Gamma(f)) f^{\beta-1}+\frac{\beta(\beta+1)(\beta+2)}{4(m-4)(m-2)} f^{\beta-1} \Delta(f) \Gamma(f) \\
\quad+\frac{(\beta-1) \beta(\beta+1)(\beta+2)}{4(m-4)(m-2)} f^{\beta-2} \Gamma(f)^{2}+\frac{(\beta+1)(\beta+2)}{4(m-4)(m-2)} f^{\beta} \Delta \Gamma(f) .
\end{gathered}
$$

Expanding the term $\Delta^{2}\left(f^{\beta+2}\right)$ (recall that $\beta=\frac{2-m+d}{m-d}$ ), after a bit of algebra, we finally get,

$$
\Gamma_{2}(f) \geqslant \frac{\beta+1}{d(\beta+1)-2 \beta}(\Delta f)^{2}-\beta \frac{\Gamma(f, \Gamma(f))}{f}-\frac{\beta(\beta-1)}{2} \frac{\Gamma(f)^{2}}{f^{2}} .
$$

First, observe that if $\beta=0$ that is $m=d+2$ then the previous inequality becomes

$$
\Gamma_{2}(f) \geqslant \frac{1}{d}(\Delta f)^{2}
$$

in others words we recover the $C D(0, d)$ condition of the Laplacian $\Delta$. 
It is more subtle to notice that, for any $\beta \in(-1,0)$ fixed, inequality (33) implies the same condition $C D(0, d)$. It is more tricky since, taking $f=1+\varepsilon g$ with $\varepsilon \rightarrow 0$, then (33) already implies $\Gamma_{2}(g) \geqslant \frac{\beta+1}{d(\beta+1)-2 \beta}(\Delta g)^{2}$ which is the $C D\left(0, \frac{d(\beta+1)-2 \beta}{\beta+1}\right)$ condition, weaker than the $C D(0, d)$ condition, since $\frac{d(\beta+1)-2 \beta}{\beta+1} \geqslant d$. The way to recover the $C D(0, d)$ condition is to replace $f$ by $\Phi(f)$ in (33), where $\Phi$ is a smooth function.

The diffusion properties in (33) one has,

$$
\begin{aligned}
\Phi^{\prime 2}\left(\Gamma_{2}(f)-a(\Delta f)^{2}\right)+\Phi^{\prime \prime} \Gamma(f)^{2}(1-a)-c \frac{\Phi^{\prime 4}}{\Phi^{2}} \Gamma(f)^{2} & +\Phi^{\prime} \Phi^{\prime \prime}(\Gamma(f, \Gamma(f))-2 a \Delta f \Gamma(f)) \\
& -2 b \frac{\Phi^{\prime 2} \Phi^{\prime \prime}}{\Phi} \Gamma(f)^{2}-b \frac{\Phi^{\prime 3}}{\Phi} \Gamma(f, \Gamma(f)) \geqslant 0,
\end{aligned}
$$

where $a=\frac{\beta+1}{d(\beta+1)-2 \beta}, b=-2 \beta$ and $c=-\beta(\beta-1)$ and for simplicity we omit the variable $f$ in the function $\Phi$. It implies that the quadratic form with respect to the variables $\Phi^{\prime}, \Phi^{\prime 2} / \Phi$ and $\Phi^{\prime \prime}$ is positive, then the determinant of its matrix is positive. We get

$$
\begin{aligned}
\Gamma_{2}(f) \geqslant \frac{1}{d}(\Delta f)^{2}+\left(\frac{-a c-a^{2}}{c(a-1)-b^{2}}-\frac{1}{d}\right)(\Delta f)^{2}+\frac{-b^{2}-c-b^{2} a}{4\left(c(a-1)-b^{2}\right)}\left(\frac{\Gamma(f, \Gamma(f))}{\Gamma(f)}\right)^{2} & +\frac{b^{2}+a c}{c(a-1)-b^{2}} \frac{\Gamma(f, \Gamma(f))}{\Gamma(f)} \Delta f .
\end{aligned}
$$

After an unpleasant computation, the inequality is independent of $\beta$, and may be written as follow

$$
\Gamma_{2}(f) \geqslant \frac{1}{d}(\Delta f)^{2}+\frac{d}{d-1}\left(\frac{\Gamma(f, \Gamma(f))}{2 \Gamma(f)}-\frac{1}{d} \Delta f\right)^{2},
$$

which is a reinforced $C D(0, d)$ condition.

Indeed, in all these computations, we could have replaced the Laplace operator $\Delta$ in $\mathbb{R}^{d}$ by any operator satisfying the $C D(0, d)$ condition. Then we could have constructed the associated $Q_{t}^{(m)}$ operator through (21), and obtain also a similar family of Beckner inequalities for this $Q_{t}^{(m)}$, still equivalent to the starting $C D(0, d)$ condition. The main difference in the Euclidean case is that then the family of inequalities for $Q_{t}^{(m)}(x)$ reduces one for $Q_{1}^{(m)}(0)$, through dilations and translations.

\section{3 $\Phi$-entropy inequalities for Cauchy type distribution}

We can extend Beckner's inequalities to a general $\Phi$-entropy inequality. For any convex function $\Phi$ on an interval $I \subset \mathbb{R}$, let us define the $\Phi$-entropy for measure $\mu$ (or a kernel) and a function $f$ on $I$,

$$
\operatorname{Ent}_{\mu}^{\Phi}(f)=\int \Phi(f) d \mu-\Phi\left(\int f d \mu\right) .
$$

Section 4.1 can be easily generalized to $\Phi$-entropy.

Definition 16 (n-Admissible functions) Let $\Phi: I \mapsto \mathbb{R}$ be a smooth function on an interval $I \subset \mathbb{R}$ and let $n<0$. We say that $\Phi$ is $n$-admissible if $\Phi^{\prime \prime}>0$ on $I$ and $\left(\Phi^{\prime \prime}\right)^{\frac{2-n}{n}}$ is concave.

In other words, $\Phi$ is n-admissible if and only if $\Phi^{\prime \prime}>0$ and moreover

$$
2 \frac{n-1}{n}\left(\Phi^{(3)}\right)^{2} \leq \Phi^{\prime \prime} \Phi^{(4)},
$$


that is $\theta=\Phi^{\prime \prime}$ satisfies condition (13).

When $n$ goes to $-\infty$, an $(-\infty)$-admissible is just a function $\Phi$ such that, $\Phi^{\prime \prime}>0$ on $I$ and $\left(1 / \Phi^{\prime \prime}\right)$ is concave. Many $\Phi$-entropy inequalities have been proved for such function $\varphi$ under the curvature-dimension condition $C D(\rho, \infty)$ (see [Cha04, BG10]). In our computations, the case $n=-\infty$ is then similar to the case $n=+\infty$.

We can state the following result.

Theorem 17 ( $\Phi$-entropy inequality for $\left.Q_{t}^{(m)}\right)$ For any function $\Phi(d-m+2)$-admissible on an interval $I$, the following holds, for any $m \geqslant d+2$,

$$
\operatorname{Ent}_{Q_{t}^{(m)}}^{\Phi}(f) \leq \frac{t^{2}}{2(m-2)} Q_{t}^{(m-2)}\left(\Phi^{\prime \prime}(f) \Gamma(f)\right),
$$

for any smooth function $f$ on $I$.

The inequality is optimal in the sense that if inequality (34) holds for a some function $\Phi$, the constant $\frac{t^{2}}{2(m-2)}$ is the optimal one.

Corollary 18 ( $\Phi$-entropy inequality for Cauchy distribution) For any function $\Phi(d-$ $m+2$ )-admissible on an interval $I$, the following holds, for $b \geqslant d+1$

$$
\operatorname{Ent}_{\nu_{b}}^{\Phi}(f) \leq \frac{1}{4(b-1)} \int \Phi^{\prime \prime}(f) \Gamma(f)\left(1+|x|^{2}\right) d \nu_{b},
$$

for any smooth function $f$ on I. The inequality is optimal.

The proof is the same as the one of Theorem 11, using the sub-harmonicity inequality (14), so we skip it, the optimality being proved in a same way.

\section{A Beckner-type inequality on the sphere}

In this section we are looking for the link between the family of Beckner inequalities (12) for $\nu_{b}$ (or $Q_{t}^{(m)}$ ) and a family of Beckner inequalities on the sphere. In all this section we assume that $d \geqslant 2$.

The unit sphere $\mathbb{S}^{d} \subset \mathbb{R}^{d+1}$ can be seen in $\mathbb{R}^{d}$ through the stereographic projection, with the carré du champ operator on the sphere

$$
\Gamma_{\mathbb{S}}(f)=\frac{\rho^{4}}{4} \Gamma(f)
$$

where $\rho(x)=\sqrt{1+|x|^{2}}$ and the spherical measure

$$
\mu_{\mathbb{S}}(d x)=\frac{1}{c(d, d)} \frac{1}{\rho^{2 d}} d x,
$$

where the normalization constant $c(d, d)$ has been defined in (16) (see [BGL14, Sec 2.2]). The Laplace-Beltrami operator takes the form in the stereographic projection representation

$$
\Delta_{\mathbb{S}}=\frac{\left(1+|x|^{2}\right)^{2}}{4} \Delta-\frac{d-2}{2}\left(1+|x|^{2}\right) \sum_{i=1}^{d} x_{i} \partial_{i},
$$


where $\Delta$ is the classical Laplacian in $\mathbb{R}^{d}$. The operator is symmetric in $L^{2}\left(\mu_{\mathbb{S}}\right)$.

It is interesting to recall that the map $\mathbb{S}^{d} \ni x \mapsto x_{d+1}$ is a eigenvector associated to the eigenvalue $-d$. This function, from the stereographic projection, takes the form

$$
u(x)=\frac{1-|x|^{2}}{1+|x|^{2}}, x \in \mathbb{R}^{d} .
$$

Then, $u$ satisfies $\Delta_{\mathbb{S}} u=-d u$ and $\Gamma_{\mathbb{S}^{d}}(u)=1-u^{2}$ since,

$$
\Gamma_{\mathbb{S}^{d}}(u)=\frac{\left(1+|x|^{2}\right)^{2}}{4} \Gamma\left(\frac{1-|x|^{2}}{1+|x|^{2}}\right)=\frac{1}{\left(1+|x|^{2}\right)^{2}} \Gamma\left(|x|^{2}\right)=\frac{4|x|^{2}}{\left(1+|x|^{2}\right)^{2}}=1-u^{2} .
$$

Indeed this computation is much easer (and indeed completely elementary) using the standard representation in the sphere, see [BGL14, Sec 2.2].

We can now transform the Beckner inequality for $Q_{t}^{(m)}$ into a new Beckner inequality on the sphere. With these new notations, inequality (26) for $t=1$ and $x=0$ becomes for smooth and nonnegative function $f$,

$$
\begin{aligned}
\frac{c(d, d)}{c(m, d)} \int f^{\beta+2} \rho^{d-m} d \mu_{\mathbb{S}}-\left(\frac{c(d, d)}{c(m, d)} \int f \rho^{d-m} d \mu_{\mathbb{S}}\right)^{\beta+2} & \\
\leq & \frac{2(\beta+1)(\beta+2)}{m-2} \frac{c(d, d)}{c(m-2, d)} \int f^{\beta} \Gamma_{\mathbb{S}}(f) \rho^{d-m-2} d \mu_{\mathbb{S} .}
\end{aligned}
$$

We concentrate to the case $\beta=\frac{d+2-m}{m-d}$, the limit of the admissible interval. Let $f=\rho^{m-d} g$ and we compute the various terms. First, we have form diffusion properties of $\Gamma_{\mathbb{S}}$,

$$
\begin{aligned}
f^{\beta} \Gamma_{\mathbb{S}}(f) \rho^{d-m-2}=g^{\beta} \rho^{2(d-m)} & \Gamma_{\mathbb{S}}\left(\rho^{m-d} g\right)= \\
& g^{\beta} \Gamma_{\mathbb{S}}(g)+2(m-d) g^{\beta+1} \Gamma_{\mathbb{S}}(\log \rho, g)+(m-d)^{2} g^{\beta+2} \Gamma_{\mathbb{S}}(\log \rho),
\end{aligned}
$$

so that

$$
\begin{aligned}
& \int f^{\beta} \Gamma_{\mathbb{S}}(f) \rho^{d-m-2} d \mu_{\mathbb{S}}= \\
& \quad \int g^{\beta} \Gamma_{\mathbb{S}}(g) d \mu_{\mathbb{S}}+(m-d) \int \frac{2}{\beta+2} \Gamma_{\mathbb{S}}\left(\log \rho, g^{\beta+2}\right) d \mu_{\mathbb{S}}+(m-d)^{2} \int g^{\beta+2} \Gamma_{\mathbb{S}}(\log \rho) d \mu_{\mathbb{S}} .
\end{aligned}
$$

With an integration by parts, we get

$$
\int \Gamma_{\mathbb{S}}\left(\log \rho, g^{\beta+2}\right) d \mu_{\mathbb{S}}=-\int g^{\beta+2} \Delta_{\mathbb{S}} \log \rho d \mu_{\mathbb{S}}
$$

and then

$$
\int f^{\beta} \Gamma_{\mathbb{S}}(f) \rho^{d-m-2} d \mu_{\mathbb{S}}=\int g^{\beta} \Gamma_{\mathbb{S}}(g) d \mu_{\mathbb{S}}+\int g^{\beta+2} K d \mu_{\mathbb{S}}
$$

where

$$
K=(m-d)^{2}\left(2 \frac{\Delta_{\mathbb{S}} \log \rho}{d-m-2}+\Gamma_{\mathbb{S}}(\log \rho)\right)
$$

since $\beta+2=(d-m-2) /(d-m)$. Secondly we have

$$
\int f^{\beta+2} \rho^{d-m} d \mu_{\mathbb{S}}=\int g^{\beta+2} \rho^{2} d \mu_{\mathbb{S}}
$$


and

$$
\int f \rho^{d-m} d \mu_{\mathbb{S}}=\int g d \mu_{\mathbb{S}}
$$

Then, inequality (36) can be written as

$$
\begin{aligned}
& \frac{c(d, d)}{c(m, d)} \int g^{\beta+2} \rho^{2} d \mu_{\mathbb{S}}-\left(\frac{c(d, d)}{c(m, d)} \int g d \mu_{\mathbb{S}}\right)^{\beta+2} \\
& \quad \leq \frac{2(\beta+1)(\beta+2)}{m-2} \frac{c(d, d)}{c(m-2, d)}\left(\int g^{\beta} \Gamma_{\mathbb{S}}(g) d \mu_{\mathbb{S}}+\int g^{\beta+2} K d \mu_{\mathbb{S}}\right),
\end{aligned}
$$

or

$$
\int g^{\beta+2} R d \mu_{\mathbb{S}}-\left(\frac{c(d, d)}{c(m, d)} \int g d \mu_{\mathbb{S}}\right)^{\beta+2} \leq \frac{2(\beta+1)(\beta+2)}{m-2} \frac{c(d, d)}{c(m-2, d)} \int g^{\beta} \Gamma_{\mathbb{S}}(g) d \mu_{\mathbb{S}}
$$

with

$$
R=\frac{c(d, d)}{c(m, d)} \rho^{2}-\frac{2(\beta+1)(\beta+2)}{m-2} \frac{c(d, d)}{c(m-2, d)} K .
$$

From (18), the definition of $\beta$ and the fact that $\rho^{2}=2 /(1+u)$ where $u$ has been defined in (35), we can write

$$
R=\frac{c(d, d)}{c(m, d)}\left(\frac{2}{1+u}-2 \frac{m-d+2}{(m-d)^{2}} \frac{K}{m-2+d}\right),
$$

and the inequality

$$
\int g^{\beta+2} R d \mu_{\mathbb{S}}-\left(\frac{c(d, d)}{c(m, d)} \int g d \mu_{\mathbb{S}}\right)^{\beta+2} \leq \frac{4(m-d+2)}{(m-d)^{2}(m-2+d)} \frac{c(d, d)}{c(m, d)} \int g^{\beta} \Gamma_{\mathbb{S}}(g) d \mu_{\mathbb{S}}
$$

We need now to compute $\Delta_{\mathbb{S}} \log \rho$ and $\Gamma_{\mathbb{S}}(\log \rho)$. We have

$$
\begin{aligned}
\Delta_{\mathbb{S}}(\log \rho)=\frac{1}{2} \Delta_{\mathbb{S}} \log \rho^{2}=-\frac{1}{2} \Delta_{\mathbb{S}} \log (1+u)=\frac{d}{2(1+u)} u & +\frac{1}{2(1+u)^{2}} \Gamma_{\mathbb{S}^{d}}(u) \\
& =\frac{d}{2(1+u)} u+\frac{1}{2(1+u)^{2}}\left(1-u^{2}\right) .
\end{aligned}
$$

Then, finally

$$
\Delta_{\mathbb{S}}(\log \rho)=\frac{1+u(d-1)}{2(1+u)}
$$

and

$$
\Gamma_{\mathbb{S}}(\log \rho)=\frac{1-u}{4(1+u)}
$$

If we plug those values on the definition of $K$, then a miracle occurs. The function $R$ is constant,

$$
R=\frac{c(d, d)}{c(m, d)} \frac{3 d+m-2}{d+m-2} .
$$

Setting now $f^{2}=g^{\beta+2}=g^{\frac{d-m-2}{d-m}}$, we obtain 
Theorem 19 (Beckner-type inequalities on the sphere) For any smooth nonnegative function $f$ on the sphere $\mathbb{S}^{d}$, and $m \geqslant d+2$, we have

$$
\int f^{2} d \mu_{\mathbb{S}} \leq A\left(\int f^{2 / p} d \mu_{\mathbb{S}}\right)^{p}+\frac{16}{(m+2-d)(3 d-2+m)} \int \Gamma_{\mathbb{S}}(f) d \mu_{\mathbb{S}}
$$

where

$$
p=1+\frac{2}{m-d} \in(1,2]
$$

and

$$
A=\left(\frac{c(d, d)}{c(m, d)}\right)^{\frac{2}{m-d}} \frac{m+d-2}{m+3 d-2} .
$$

\section{Remarks 20}

- The function $R$ is constant only for the parameter $p=1+\frac{2}{m-d}$.

- When $m>d+2, A>1$. In that case inequality (37) is not tight, in the sense that when $g=1$, we do not have an equality. But the inequality is still optimal since it is saturated for $f=\rho^{\frac{d-m-2}{2}}$.

- When $m=d+2$, then $A=1, p=2$ and inequality (37) is nothing else than the optimal Poincaré inequality on the sphere,

$$
\int f^{2} d \mu_{\mathbb{S}}-\left(\int f^{2} d \mu_{\mathbb{S}}\right)^{2} \leq \frac{1}{d} \int \Gamma_{\mathbb{S}}(f) d \mu_{\mathbb{S}}
$$

- The classical Beckner inequalities hold for the spherical model (see e.g. [BGL14, Rmk 6.8.4]): for any $p \in(1,2]$

$$
\int f^{2} d \mu_{\mathbb{S}} \leq\left(\int|f|^{2 / p} d \mu_{\mathbb{S}}\right)^{p}+\frac{p-1}{p d} \int \Gamma_{\mathbb{S}}(f) d \mu_{\mathbb{S}}
$$

Inequality (38) is optimal in the sense that $\frac{p-1}{p d}$ is the best constant. Only constant functions saturate inequality $(38)$ for $p \in(1,2)$. And when $p$ goes to 1 , inequality (38) provides the Logarithmic Sobolev inequality on the sphere

$$
\int f^{2} \log \frac{f^{2}}{\int f^{2} d \mu_{\mathbb{S}}} d \mu_{\mathbb{S}} \leq \frac{2}{d} \int \Gamma_{\mathbb{S}}(f) d \mu_{\mathbb{S}}
$$

proved in [MW82] (see also [BGL14, Thm. 5.7.4]). On the other hand, this last logarithmic Sobolev inequality implies back the full family $(38), p \in(1,2]$.

Let us mention that inequality (38) has been generalized by in [DEKL14] in the following way,

$$
\Psi\left(\frac{1-\int f^{2 / p} d \mu_{\mathbb{S}}}{p-1}\right) \leq \int \Gamma_{\mathbb{S}}(f) d \mu_{\mathbb{S}},
$$

for every nonnegative function $f$ such that $\int f^{2} d \mu_{\mathbb{S}}=1$ and for some explicit function $\Psi$.

- So (37) appears as a new and optimal inequality on the sphere. It is probably worth to compare it to the del Pino-Dolbeault family of optimal Gagliardo-Nirenberg inequalities,

$$
\|f\|_{2 \frac{a-1}{a-2}} \leq C\|\nabla f\|_{2}^{\theta}\|f\|_{\frac{2 a}{a-2}}^{1-\theta}, \quad a \geqslant d,
$$

where $\theta$ is a fixed by scaling properties, see [DD02]. 
- The Taylor expansion (when $m \rightarrow \infty$ ) of the constant $A$ is given by

$$
A=1+d \frac{\log (m)}{m}+\frac{C}{m}+o(1 / m)
$$

where $C$ is a constant depending on the dimension d. This will be used in the rest of the section.

Even if we are not able from Theorem 19 to reach directly the Logarithmic Sobolev inequality, inequality (37) contains enough information to obtain a Sobolev inequality for the spherical model, however with a non optimal constant.

For this, we are going to obtain from (37) a Nash inequality on the sphere.

First, it is not difficult to see that there exits two constants $\alpha, \beta>0$ depending only on $d$, such that, for any $m \geqslant d+2$,

$$
A \leq \alpha^{\frac{2}{m-d}} m^{\frac{d}{m-d}}
$$

which is just a precise form of the previous Taylor expansion, and

$$
\frac{16}{(m+2-d)(3 d-2+m)} \leq \frac{\beta}{m^{2}} .
$$

From Hölder's inequality, if $p \in[1,2]$,

$$
\left(\int|f|^{2 / p} d \mu_{\mathbb{S}}\right)^{p} \leq\left(\int|f| d \mu_{\mathbb{S}}\right)^{p-1}\left(\int f^{2} d \mu_{\mathbb{S}}\right)^{2-p}
$$

with $p=1+2 /(m-d) \in[1,2]$. Inequality (37) becomes, for any nonnegative function $f$ such that $\int f d \mu_{\mathbb{S}}=1$, and any $m \geqslant m+2$,

$$
\int f^{2} d \mu_{\mathbb{S}} \leq \alpha^{\frac{2}{m-d}} m^{\frac{d}{m-d}}\left(\int f^{2} d \mu_{\mathbb{S}}\right)^{1-\frac{2}{m-d}}+\frac{\beta}{m^{2}} \int \Gamma_{\mathbb{S}}(f) d \mu_{\mathbb{S}} .
$$

Let define for $x \geqslant 0$, the map

$$
\varphi_{m}(x)=\alpha^{\frac{2}{m-d}} m^{\frac{d}{m-d}} x^{1-\frac{2}{m-d}}+\frac{\beta}{m^{2}} E,
$$

where $E=\int \Gamma_{\mathbb{S}}(f) d \mu_{\mathbb{S}}$. The function $\varphi_{m}$ as a unique fixed point $x_{m}>0$, and the previous inequality implies that $\int f^{2} d \mu_{\mathbb{S}} \leq x_{m}$.

Let us prove that for any $m \geqslant d+2$,

$$
x_{m} \leq \alpha m^{d / 2}\left(1+\frac{\beta E}{2 \alpha m^{1+d / 2}}\right)=a_{m} .
$$

To prove such an inequality, it is enough to prove that, for any $m \geqslant d+2, \varphi_{m}\left(a_{m}\right) \leq a_{m}$. We have

$$
\begin{aligned}
\varphi_{m}\left(a_{m}\right)= & \alpha m^{d / 2}\left(1+\frac{\beta E}{2 \alpha m^{1+d / 2}}\right)^{1-\frac{2}{m-d}}+\frac{\beta}{m^{2}} E \\
& \leq \alpha m^{d / 2}\left[\left(1+\frac{\beta E}{2 \alpha m^{1+d / 2}}\right)^{1-\frac{2}{m}}+\frac{\beta}{m^{2+\frac{d}{2}}} E\right] \leq \alpha m^{d / 2}\left(1+\frac{\beta E}{2 \alpha m^{1+d / 2}}\right)=a_{m},
\end{aligned}
$$


where we used the inequality $(1+x)^{u} \leq 1+u x$, for $x>0$ and $u \in(0,1)$. Modifying the numerical constants (depending only on $d$ ), we see that for any $m \geqslant d+2$,

$$
\int f^{2} d \mu_{\mathbb{S}} \leq C m^{d / 2}+\frac{C}{m^{d / 2}} \int \Gamma_{\mathbb{S}}(f) d \mu_{\mathbb{S}}
$$

Optimizing with respect to the parameter $m \geqslant d+2$, we have obtained, for some other constant $C>0$,

$$
\begin{aligned}
\int f^{2} d \mu_{\mathbb{S}} \leq C\left(\left(\int|f| d \mu_{\mathbb{S}}\right)^{2}+\int \Gamma_{\mathbb{S}}(f) d \mu_{\mathbb{S}}\right)^{\frac{d}{d+2}}\left(\int|f| d \mu_{\mathbb{S}}\right)^{\frac{2}{d+2}} \\
\leq C\left(\int f^{2} d \mu_{\mathbb{S}}+\int \Gamma_{\mathbb{S}}(f) d \mu_{\mathbb{S}}\right)^{\frac{d}{d+2}}\left(\int|f| d \mu_{\mathbb{S}}\right)^{\frac{2}{d+2}}
\end{aligned}
$$

from Jensen inequality.

This last inequality is a so-called Nash inequality, and is known to be equivalent to the Sobolev inequality (up to the optimal constant) for the spherical model, see [BGL14, Prop. 6.2.3],

$$
\left(\int|f|^{\frac{2 d}{d-2}} d \mu_{\mathbb{S}}\right)^{\frac{d-2}{d}} \leq C \int f^{2} d \mu_{\mathbb{S}}+C \int \Gamma_{\mathbb{S}}(f) d \mu_{\mathbb{S}}
$$

From the Poincaré inequality $(m=d+2)$ and [BGL14, Prop. 6.2.2], this inequality implies a tight Sobolev inequality. We have obtained

Theorem 21 (From Beckner inequalities to Sobolev inequality on the sphere) From the family of Beckner type inequalities (37), one can prove that there exists a constant $C>0$ depending only on $d$ such that, for any smooth functions $f$ on the sphere $\mathbb{S}^{d}$,

$$
\left(\int|f|^{\frac{2 d}{d-2}} d \mu_{\mathbb{S}}\right)^{\frac{d-2}{d}} \leq \int f^{2} d \mu_{\mathbb{S}}+C \int \Gamma_{\mathbb{S}}(f) d \mu_{\mathbb{S}} .
$$

Conclusion: The family of optimal inequalities (37) appears as a new form of optimal inequalities on the sphere, implying a Sobolev inequality.

Acknowledgements. This work was partly written while the two first authors were visiting Institut Mittag-Leffler in Stockholm; it is a pleasure for them to thank this institution for its kind hospitality. We would like also to warmly thank the referee who give interesting comments and pointing out references.

This research was supported by the French ANR-17-CE40-0030 EFI project.

\section{References}

[ABJ18] M. Arnaudon, M. Bonnefont, and A. Joulin. Intertwinings and generalized BrascampLieb inequalities. Rev. Mat. Iberoam., 34(3):1021-1054, 2018.

[AD05] A. Arnold and J. Dolbeault. Refined convex Sobolev inequalities. J. Funct. Anal., 225(2):337-351, 2005. 
[Bak94] D. Bakry. L'hypercontractivité et son utilisation en théorie des semigroupes. In Lectures on probability theory (Saint-Flour, 1992), Lecture Notes in Math. 1581, pages 1-114. Springer, Berlin, 1994.

$\left[\mathrm{BBD}^{+} 07\right]$ A. Blanchet, M. Bonforte, J. Dolbeault, G. Grillo, and J.-L. Vázquez. HardyPoincaré inequalities and applications to nonlinear diffusions. C. R. Math. Acad. Sci. Paris, 344(7):431-436, 2007.

[BCLS95] D. Bakry, T. Coulhon, M. Ledoux, and L. Saloff-Coste. Sobolev inequalities in disguise. Indiana Univ. Math. J., 44(4):1032-1074, 1995.

[BDGV10] M. Bonforte, J. Dolbeault, G. Grillo, and J. L. Vazquez. Sharp rates of decay of solutions to the nonlinear fast diffusion equation via functional inequalities. Proc. Natl. Acad. Sci. USA, 107(38):16459-16464, 2010.

[BÉ85] D. Bakry and M. Émery. Diffusions hypercontractives. In Séminaire de probabilités, XIX, 1983/84, Lecture Notes in Math. 1123, pages 177-206. Springer, Berlin, 1985.

[Bec89] W. Beckner. A generalized Poincaré inequality for Gaussian measures. Proc. Am. Math. Soc., 105(2):397-400, 1989.

[BG10] F. Bolley and I. Gentil. Phi-entropy inequalities for diffusion semigroups. J. Math. Pures Appl. (9), 93(5):449-473, 2010.

[BGL14] D. Bakry, I. Gentil, and M. Ledoux. Analysis and geometry of Markov diffusion operators. Cham: Springer, 2014.

[BJM16] M. Bonnefont, A. Joulin, and Y. Ma. A note on spectral gap and weighted Poincaré inequalities for some one-dimensional diffusions. ESAIM Probab. Stat., 20:18-29, 2016.

[BL09] S. G. Bobkov and M. Ledoux. Weighted Poincaré-type inequalities for Cauchy and other convex measures. Ann. Probab., 37(2):403-427, 2009.

[BS96] A.N. Borodin and P. Salminen. Handbook of Brownian motion - facts and formulae. Basel: Birkhäuser, 1996.

[Cha04] D. Chafaï. Entropies, convexity, and functional inequalities: on $\Phi$-entropies and $\Phi$ Sobolev inequalities. J. Math. Kyoto Univ., 44(2):325-363, 2004.

[DD02] M. Del Pino and J. Dolbeault. Best constants for Gagliardo-Nirenberg inequalities and applications to nonlinear diffusions. J. Math. Pures Appl. (9), 81(9):847-875, 2002.

[DEKL14] J. Dolbeault, M. J. Esteban, M. Kowalczyk, and M. Loss. Improved interpolation inequalities on the sphere. Discrete Contin. Dyn. Syst., Ser. S, 7(4):695-724, 2014.

[DGS18] D. Dupaigne, I. Gentil, and Zugmeyer S. A family of Beckner inequalities under different curvature-dimension conditions. 2018.

[DNS08] J. Dolbeault, B. Nazaret, and G. Savaré. On the Bakry-Emery criterion for linear diffusions and weighted porous media equations. Comm. Math. Sci., 6(2):477-494, 2008.

[IV17] P. Ivanisvili and A. Volberg. Improving Beckner's bound via Hermite functions. Anal. PDE, 10(4):929-942, 2017.

[IV18] P. Ivanisvili and A. Volberg. Isoperimetric functional inequalities via the maximum principle: the exterior differential systems approach. In 50 years with Hardy spaces, volume 261 of Oper. Theory Adv. Appl., pages 281-305. Birkhäuser/Springer, Cham, 2018 . 
[KN04] S. Kotz and S. Nadarajah. Multivariate $t$ distributions and their applications. Cambridge University Press, 2004.

[Mil17] E. Milman. Beyond traditional curvature-dimension. I: New model spaces for isoperimetric and concentration inequalities in negative dimension. Trans. Am. Math. Soc., 369(5):3605-3637, 2017.

[MW82] C. E. Mueller and F. B. Weissler. Hypercontractivity for the heat semigroup for ultraspherical polynomials and on the n-sphere. J. Funct. Anal., 48:252-283, 1982.

[Ngu14] V. H. Nguyen. Dimensional variance inequalities of Brascamp-Lieb type and a local approach to dimensional Prékopa's theorem. J. Funct. Anal., 266(2):931-955, 2014.

[Ngu18] V. H. Nguyen. Phi-entropy inequalities and asymmetric covariance estimates for convex measures. 2018. To appear in Bernoulli.

[Oht16] S-i Ohta. $(K, N)$-convexity and the curvature-dimension condition for negative $N$. J. Geom. Anal., 26(3):2067-2096, 2016.

[Øk03] B. Øksendal. Stochastic differential equations. An introduction with applications. Berlin: Springer, 6th ed. edition, 2003.

[Pav14] G. A. Pavliotis. Stochastic processes and applications. Diffusion processes, the FokkerPlanck and Langevin equations. New York, NY: Springer, 2014.

[Sch01] G. Scheffer. Inégalités fonctionnelles, géométrie conforme et noyaux markoviens. Phd, 2001.

[Sch03] G. Scheffer. Local Poincaré inequalities in non-negative curvature and finite dimension. J. Funct. Anal., 198(1):197-228, 2003. 